\title{
Decoherence of a Damped Anisotropic Harmonic Oscillator under Magnetic Field Effects in a Two-Dimensional Noncommutative Phase-Space
}

\author{
Martin Tcoffo, ${ }^{1,2}$, Germain Yinde Deuto', Issofa Nsangou1, Armel Azangue Koumetio', \\ Lylyane S. Yonya Tchapda', Alain G. Tene ${ }^{1}$ \\ ${ }^{1}$ Research Unit of Condensed Matter, Electronics and Signal Processing, University of Dschang, Dschang, Cameroon \\ ${ }^{2}$ Centre d'Etude et de Recherche en Agronomie et en Biodiversité, FASA, Université de Dschang, Dschang, Cameroun \\ Email: mtchoffo2000@yahoo.fr
}

How to cite this paper: Tcoffo, M., Yinde Deuto, G., Nsangou, I., Azangue Koumetio, A., Yonya Tchapda, L.S. and Tene, A.G. (2020) Decoherence of a Damped Anisotropic Harmonic Oscillator under Magnetic Field Effects in a Two-Dimensional Noncommutative Phase-Space. Journal of Applied Mathematics and Physics, 8, 2801-2823.

https://doi.org/10.4236/jamp.2020.812207

Received: October 27, 2020

Accepted: December 7, 2020

Published: December 10, 2020

Copyright $\odot 2020$ by author(s) and Scientific Research Publishing Inc. This work is licensed under the Creative Commons Attribution International License (CC BY 4.0).

http://creativecommons.org/licenses/by/4.0/

\begin{abstract}
In this paper, decoherence of a damped anisotropic harmonic oscillator in the presence of a magnetic field is studied in the framework of the Lindblad theory of open quantum systems in noncommutative phase-space. General fundamental conditions that should follow our quantum mechanical diffusion coefficients appearing in the master equation are kindly derived. From the master equation, the expressions of density operator, the Wigner distribution function, the expectation and variance with respect to coordinates and momenta are obtained. Based on these quantities, the total energy of the system is evaluated and simulations show its dependency to phase-space structure and its improvement due to noncommutativity effects and the environmental temperature as well. In addition, we also evaluate the decoherence time scale and show that it increases with noncommutativity phase-space effects as compared to the commutative case. It turns out from simulations that this time scale is significantly improved under magnetic field effects.
\end{abstract}

\section{Keywords}

Decoherence, Noncommutative Phase-Space, Lindblad Theory, Wigner

Distribution

\section{Introduction}

The theory of open quantum systems has become one of the main interests of physicists during the last few decades due to their strong applications in several 
domains of physics, especially in solid state physics [1], quantum optics [2], nanotechnology [3], quantum measurement theory [4], etc. Indeed, various research works conducted in the field proved that perfect isolated quantum system does not exist, since any realistic system permanently interacts with its environment, which typically presents a large number of degrees of freedom [5]. A physical system interacting with the environment is referred to an open system [6]. Thus, every physical system is affected by the presence of its surroundings leading to dissipation, thermalization and in the quantum case, decoherence [6]. Many reasons lead scientists to pay particular attention to the above mentioned phenomena, here, we refer particularly to decoherence and dissipation, which are the main obstacle in the realization of quantum computers and other quantum devices on one hand [7] [8] [9]. On the other hand, several interesting proposals to exploit the interaction between quantum systems and their environment to dissipatedly engineer challenging state of matter have emerged very recently [6].

In fact, dissipation in an open system results from microscopic reversible interactions between the observable system and the environment. It implies irreversibility and therefore, a preferred direction in time. However, we are particularly interested in this work on the phenomenon of decoherence, which somewhat "regroups dissipation" and introduces the (partial) destruction of quantum coherence through the interaction of quantum mechanical system with its surrounding [10]. From the theoretical analysis point of view, decoherence can be described with the help of microscopic models based on the interaction of quantum mechanical systems with a collection of an infinite number of harmonic oscillators, representing the environment [11] [12]. The strength and nature of the coupling with environment play a crucial role in selecting the preferred states leading to a distance-dependent nature of typical interaction. Furthermore, decoherence simply characterizes the transition of a given quantum system to classical one due to interactions, thus, the particular time that a quantum system starts behaving classically is known as "decoherence time" [12] [13] [14]. Whereas, the decoherence time scale defines the time during which quantum interferences between quantum states disappear (i.e. loss of quantum properties) [13] [15]. Deep studies have been made to determine this particular time and to see whether or not it can be controlled due to its relevance in many interesting physical problems such as quantum computation and quantum information processing [16], heavy ion collisions [17], etc. In many cases, physicists are also interested in understanding the specific causes of quantum decoherence in order to prevent it from damaging quantum states and protect the information stored inside [18]. Thus, it was discovered that, for most of superposed quantum states, the associated decoherence time is often found to be smaller than the corresponding relaxation (damping) time. Several research works have been successfully investigated to determine this time in the literature, many of them found that the main tool for its investigation is the master equations (ME) dynamics approach, which describes the evolution in time of the density operator 
of a given particle. The ME approach allows describing various physical phenomena such as entanglement [19], decoherence and quantum to classical transition [20]. The most general ME approach was developed by Lindblad [5] [19] [21].

Indeed, the Lindblad formalism ME approach has been used to solve many concrete theoretical two modes systems and applied to various physical phenomena, for instance, in treatments of damped collective modes, in deep inelastic ions collisions, in nuclear physics [22] [23], or in the description of quantum dissipation in one dimensional harmonic oscillator [16]. But almost all the above studies have been conducted considering a commutative phase-space (i.e. the space-time coordinates and momenta can be measured simultaneously according to the Heisenberg principle). However, it was demonstrated that, the above mentioned problems including decoherence, could be treated in a noncommuting phase-space with significant results. For this reason, Dragovich et al. [24] investigated the influence of noncommutativity (NCty) on the occurrence of the so-called decoherence effect with external magnetic field, and they found that the decoherence can be highly affected when the external control (magnetic field) is constrained by the noncommutative (NC) effects. Ghorashi et al. [25] discovered that, by modifying the master equation of two-dimensional harmonic oscillators under the Brownian motion, the NCty effects lead to increase the rate of decoherence. Moreover, Tchoffo et al. [26] in their investigation on kinematical Brownian motion of harmonic oscillator in NC space showed that the structure of Fokker Planck's equation is not modified (i.e. the factorization theorem is conserved in both commutative and NC space). In the same idea, Santos et al. [27] found that, NCty induces a non-vanishing correlation between both coordinates at different times. Very recently in our previous works on decoherence of quantum Brownian particle in NC space, we realized that the NCty effects intervene in the system as a disruption and therefore, increase the rate of decoherence in the system [28]. Although significant results were achieved in the above mentioned works, they usually considered only the space coordinates as NC and the question that emerges is to know what could happen if beside coordinates the momentum was also NC. An answer to this question is the main objective of this research paper, with the approach consisting of deriving the decoherence time scale of our system in NC phase-space by the Lindblad theory for open quantum systems in NC phase-space approach.

To achieve this objective, we structure the paper as follows. In Section 2, we present a general model of the system under magnetic field effects in NC phase-space. We review the Lindblad ME approach of the density operator of the considered open system in the same section. The dynamical equation of the covariance matrix associated to the system state is derived in Section 3. We determine in Section 4 the density matrix and the Wigner function of the considered system. Section 5 is devoted to our main results where therein the decoherence time scale and the total energy of the considered system in NC phase-space are 
derived. Finally Section 6 presents some concluding remarks.

\section{Model Hamiltonian}

The model described in this work forms the standard pattern of a damped quantum harmonic oscillator in NC phase-space. Similar model was already introduced in the literature [29] [30] [31] [32] [33], but with different approach and for different motivations. For instance, in [30] it was used to analyze the Landau diamagnetism in NC space, while in [32] it was introduced to study the connection between dissipation and NCty. However, in this work our oscillator is assumed to be a non-relativistic charged particle of mass $m$ moving in a two-dimensional NC phase-space under the effect of a homogeneous magnetic field, and confined by a damped anisotropic harmonic potential with frequencies $\omega_{1}$ and $\omega_{2}$ in the $x$ and $y$ directions respectively. The Hamiltonian of this system is given by:

$$
H=\frac{1}{2 m}\left(\hat{p}_{1}-\frac{e}{c} \hat{A}_{1}\right)^{2}+\frac{1}{2 m}\left(\hat{p}_{2}-\frac{e}{c} \hat{A}_{2}\right)^{2}+\frac{m}{2}\left(\omega_{1}^{2} \hat{q}_{1}^{2}+\omega_{2}^{2} \hat{q}_{2}^{2}\right)+k_{12} \hat{q}_{1} \hat{q}_{2},
$$

where $\left(\hat{A}_{1}, \hat{A}_{2}\right)$ are the components of the potential vector of the magnetic field, $k_{12}$ the damping parameter, $\left(\hat{q}_{1}, \hat{q}_{2}\right)$ and $\left(\hat{p}_{1}, \hat{p}_{2}\right)$ are respectively the noncommutative coordinates and momenta of the quantum oscillator satisfying the following commutation relations [34] [35] [36] [37] [38] $\left[\hat{q}_{k}, \hat{q}_{l}\right]=i \theta \varepsilon_{k l}$, $\left[\hat{p}_{k}, \hat{p}_{l}\right]=i \eta \varepsilon_{k l},\left[\hat{q}_{k}, \hat{p}_{l}\right]=i \tilde{\hbar} \delta_{k l}$, with $\tilde{\hbar}=\hbar\left(1+\frac{\theta \eta}{4 \hbar^{2}}\right)$ the effective Planck constant, $\theta$ and $\eta$ the NC parameters with respect to the coordinates and momenta, respectively. Due to the fact that the NCty structure of the phase-space is a pure geometrical property, its physical effects are independent of the particle nature. Therefore, NCty between momenta arises naturally as a consequence of NC coordinates, since momenta are defined to be partial derivatives of the action with respect to the NC coordinates. The NC parameters $\theta$ and $\eta$ have the dimensions of (length) ${ }^{2}$ and (momentum) $)^{2}$ respectively [35] [39]. The NC coordinates and momenta in terms of the commuting ones are defined as:

$$
\left\{\begin{array}{l}
\hat{q}_{1}=q_{1}-\frac{\theta}{2 \hbar} p_{2}, \hat{q}_{2}=q_{2}+\frac{\theta}{2 \hbar} p_{1} \\
\hat{p}_{1}=p_{1}+\frac{\eta}{2 \hbar} q_{2}, \hat{p}_{2}=p_{2}-\frac{\eta}{2 \hbar} q_{1}
\end{array}\right.
$$

where the coordinates $q_{k}$ and momenta $p_{k}$ obey the usual commutation relations $\left[q_{k}, q_{l}\right]=0,\left[p_{k}, p_{l}\right]=0$ and $\left[q_{k}, p_{l}\right]=i \hbar \delta_{k l}, k, l=1,2$. By the gauge symmetric relation, the vector potentials of the magnetic field $\hat{A}_{k}$ can be defined as:

$$
\hat{A}=\left(-\frac{B}{2} \hat{q}_{2}, \frac{B}{2} \hat{q}_{1}\right)
$$

Using the latest relation and Equation (2), the Hamiltonian (1) can be rewrit- 
ten as follows:

$$
\begin{aligned}
H= & \frac{1}{2 m_{1}} p_{1}^{2}+\frac{1}{2 m_{2}} p_{2}^{2}+\frac{m}{2}\left(\varpi_{1}^{2} q_{1}^{2}+\varpi_{2}^{2} q_{2}^{2}\right) \\
& +k_{12}\left(q_{1} q_{2}+\frac{\theta}{2 \hbar}\left(q_{1} p_{1}-q_{2} p_{2}\right)-\frac{\theta^{2}}{2 \hbar^{2}} p_{1} p_{2}\right) \\
& -\left(\lambda_{1} q_{1} p_{2}-\lambda_{2} q_{2} p_{1}\right)
\end{aligned}
$$

where

$$
\begin{aligned}
& \left\{\begin{array}{l}
\varpi_{1}=\sqrt{\omega_{1}^{2}+\frac{\omega_{c}^{2}}{4}+\frac{\omega_{c} \eta}{2 m \hbar}+\frac{\eta^{2}}{4 m^{2} \hbar^{2}}} \\
\varpi_{2}=\sqrt{\omega_{2}^{2}+\frac{\omega_{c}^{2}}{4}+\frac{\omega_{c} \eta}{2 m \hbar}+\frac{\eta^{2}}{4 m^{2} \hbar^{2}}},
\end{array}\right. \\
& \left\{\begin{array}{l}
\lambda_{1}=\frac{m \theta}{2 \hbar}\left(\omega_{1}^{2}+\frac{\omega_{c}^{2}}{4}\right)+\frac{\omega_{c}}{2}\left(1+\frac{\theta \eta}{4 \hbar^{2}}\right)+\frac{\eta}{2 m \hbar}, \\
\lambda_{2}=\frac{m \theta}{2 \hbar}\left(\omega_{1}^{2}+\frac{\omega_{c}^{2}}{4}\right)+\frac{\omega_{c}}{2}\left(1+\frac{\theta \eta}{4 \hbar^{2}}\right)+\frac{\eta}{2 m \hbar},
\end{array}\right.
\end{aligned}
$$

with $m_{1}=\frac{m}{\left(1+\frac{m \omega_{c} \theta}{4 \hbar}\right)^{2}+\left(\frac{m \theta \omega_{2}}{2 \hbar}\right)^{2}}$ and $m_{2}=\frac{m}{\left(1+\frac{m \omega_{c} \theta}{4 \hbar}\right)^{2}+\left(\frac{m \theta \omega_{1}}{2 \hbar}\right)^{2}}$. It is important to notice that the effective masses $m_{1}$ and $m_{2}$ are different due to the anisotropy of the system, and $\omega_{c}=\frac{e B}{m c}$ is the cyclotron frequency. The last term appearing in the Hamiltonian (4) is due to the NCty effects. This term introduces some difficulties to solve the eigenvalue equation of the corresponding Hamiltonian, thus the whole Hamiltonian requires further transformations. For this reason, let's define a canonical transformation involving the set of coordinates-momenta $\left(q_{1}, q_{2}, p_{1}, p_{2}\right)$ [31] [32] [40] by:

$$
\left\{\begin{array} { l } 
{ q _ { 1 } = \chi \operatorname { c o s } ( \Phi ) Q _ { 1 } + \frac { 1 } { \rho } \operatorname { s i n } ( \Phi ) P _ { 2 } , } \\
{ q _ { 2 } = \chi \operatorname { c o s } ( \Phi ) Q _ { 2 } + \frac { 1 } { \rho } \operatorname { s i n } ( \Phi ) P _ { 1 } , }
\end{array} \text { and } \left\{\begin{array}{l}
p_{1}=-\rho \sin (\Phi) Q_{2}+\frac{1}{\chi} \cos (\Phi) P_{1}, \\
p_{2}=-\rho \sin (\Phi) Q_{1}+\frac{1}{\chi} \cos (\Phi) P_{2} .
\end{array}\right.\right.
$$

The new coordinates $\left(Q_{1}, Q_{2}\right)$ and momenta $\left(P_{1}, P_{2}\right)$ also satisfy the canonical commutation relations:

$$
\left[Q_{1}, P_{1}\right]=\left[Q_{2}, P_{2}\right]=i \hbar,
$$

while all other permutations vanish. Using the transformations of Equation (6), the Hamiltonian (4) becomes:

$$
\begin{aligned}
H= & \frac{1}{2 M_{1}} P_{1}^{2}+\frac{1}{2 M_{2}} P_{2}^{2}+\frac{M_{1}}{2} \Omega_{1}^{2} Q_{1}^{2}+\frac{M_{2}}{2} \Omega_{2}^{2} Q_{2}^{2}+c_{12} Q_{1} Q_{2} \\
& +\mu_{11} Q_{1} P_{2}+\mu_{22} Q_{2} P_{2}+d_{12} P_{1} P_{2},
\end{aligned}
$$

where

$$
M_{1}=\frac{1}{2 I_{1}^{2}}, M_{2}=\frac{1}{2 I_{2}^{2}}, \Omega_{1}=2 I_{1} K_{1}, \Omega_{2}=2 I_{2} K_{2},
$$

with 


$$
\left\{\begin{array}{l}
I_{1}^{2}=\frac{1}{2 \rho^{2}}\left[\frac{\rho^{2}}{m_{1} \chi^{2}} \cos ^{2}(\Phi)+m \varpi_{2}^{2} \sin ^{2}(\Phi)+\frac{\lambda_{2} \rho}{\chi} \sin (2 \Phi)\right], \\
I_{2}^{2}=\frac{1}{2 \rho^{2}}\left[\frac{\rho^{2}}{m_{2} \chi^{2}} \cos ^{2}(\Phi)+m \varpi_{1}^{2} \sin ^{2}(\Phi)+\frac{\lambda_{1} \rho}{\chi} \sin (2 \Phi)\right], \\
K_{1}^{2}=\frac{\chi^{2}}{2}\left[\frac{\rho^{2}}{m_{2} \chi^{2}} \sin ^{2}(\Phi)+m \varpi_{1}^{2} \cos ^{2}(\Phi)+\frac{\lambda_{1} \rho}{\chi} \sin (2 \Phi)\right], \\
K_{2}^{2}=\frac{\chi}{2}\left[\frac{\rho^{2}}{m_{1} \chi^{2}} \sin ^{2}(\Phi)+m \varpi_{2}^{2} \cos ^{2}(\Phi)+\frac{\lambda_{2} \rho}{\chi} \sin (2 \Phi)\right],
\end{array}\right.
$$

and

$$
\begin{aligned}
& \left\{\begin{array}{l}
\mu_{11}=k_{12} \frac{\rho}{2 \chi}\left[\left(\frac{\chi^{2}}{\rho^{2}}+\frac{\theta^{2}}{4 \hbar^{2}}\right) \sin (2 \Phi)+\frac{\theta \chi}{\hbar \rho}\right], \\
\mu_{22}=k_{12} \frac{\rho}{2 \chi}\left[\left(\frac{\chi^{2}}{\rho^{2}}+\frac{\theta^{2}}{4 \hbar^{2}}\right) \sin (2 \Phi)-\frac{\theta \chi}{\hbar \rho}\right],
\end{array}\right. \\
& \left\{\begin{array}{l}
c_{12}=k_{12} \chi^{2}\left[\cos ^{2}(\Phi)-\frac{\theta^{2} \rho^{2}}{4 \hbar^{2} \chi^{2}} \sin ^{2}(\Phi)\right], \\
d_{12}=\frac{k_{12}}{\rho^{2}}\left[\sin ^{2}(\Phi)-\frac{\theta^{2} \rho^{2}}{4 \hbar^{2} \chi^{2}} \cos ^{2}(\Phi)\right] .
\end{array}\right.
\end{aligned}
$$

The set $(\rho, \chi, \Phi)$ satisfies the following relations:

$$
\left\{\begin{array}{l}
\frac{\rho}{\chi}=\sqrt{\frac{m m_{1} m_{2}\left(\lambda_{2} \varpi_{1}^{2}+\lambda_{1} \varpi_{2}^{2}\right)}{m_{2} \lambda_{1}+m_{1} \lambda_{2}}} \\
\tan (2 \Phi)=\frac{2 \sqrt{m_{1} m_{2}\left(m_{1} \lambda_{2}+m_{2} \lambda_{1}\right)\left(\lambda_{2} \varpi_{1}^{2}+\lambda_{1} \varpi_{2}^{2}\right)}}{\sqrt{m}\left(m_{2} \varpi_{1}^{2}-m_{1} \varpi_{2}^{2}\right)}
\end{array}\right.
$$

Equation (8) is the Hamiltonian of a harmonic oscillator in two-dimensional NC phase-space, where the mass varies in terms of the NC parameters, which can provide simple solution to the eigenvalues equation. Table 1 depicts the values of all the parameters and constants that appear in this Hamiltonian and in the entire work, and which will be necessary for numerical simulations in Section 5. In order to investigate the dynamics of two bosonic modes (Harmonic oscillators) in weak interaction with thermal reservoir in NC phase-space, we employ the axiomatic formalism based on the completely positive quantum dynamical semigroups [41].

\section{Derivation of the Variance and Covariance Matrices}

Generally, the simplest dynamics for an open system which describes an irreversible time evolution is given by the Gorini-Kossakowski-Linblad-Sudarshan (GKLS) quantum master equation [41] [42]. Indeed, the GKLS method is based on the non-Markovian approximation, meaning that there are no memory effects. The GKLS quantum non-Markovian master equation for the density operator $\rho(t)$ in the Schrodinger representation is given [42] [43] [44] by: 
Table 1. Recapitulation of all the parameters and constants that are used in this work.

\begin{tabular}{|c|c|c|c|}
\hline Parameter & Symbol & Value & Unit \\
\hline Charge of electron & $e$ & 1 & $\mathrm{C}$ \\
\hline Magnetic field & $B$ & $\propto 10^{-2}$ & $\mathrm{~T}$ \\
\hline Reduced Planck constant & $\hbar$ & 1 & $\mathrm{j} \cdot \mathrm{s}$ \\
\hline Frequency in the $\mathrm{x}$-direction & $\omega_{1}$ & 1.5 & $\mathrm{~s}^{-1}$ \\
\hline Frequency in the y-direction & $\omega_{2}$ & 2.5 & $\mathrm{~s}^{-1}$ \\
\hline Effective mass & $m$ & 1 & $\mathrm{~kg}$ \\
\hline Light constant & $c$ & 1 & $\mathrm{~m} \cdot \mathrm{s}^{-1}$ \\
\hline Squeezing parameter & $\delta$ & 0.5 & $\mathrm{~s}^{-1}$ \\
\hline Friction coefficients & $\alpha_{k l}, \quad \beta_{k l}, \quad \lambda_{k l}$ & between 0 and 1 & dimensionless \\
\hline Boltzmann constant & $k$ & 1 & $\mathrm{~m}^{2} \cdot \mathrm{kg} \cdot \mathrm{s}^{-2} \cdot \mathrm{K}^{-1}$ \\
\hline Temperature & $T$ & between 0 and 20 & $\mathrm{~K}$ \\
\hline NC-parameter along the coordinates & $\theta$ & $\propto 10^{-38}$ & $\mathrm{~m}^{2}$ \\
\hline NC-parameter along the momentum & $\eta$ & $\propto 10^{-60}$ & $\mathrm{~kg}^{2} \cdot \mathrm{m}^{2} \cdot \mathrm{s}^{-2}$ \\
\hline
\end{tabular}

$$
L(\rho)=\frac{\mathrm{d} \rho}{\mathrm{d} t}=-\frac{i}{\hbar}[H, \rho]+\frac{1}{2 \hbar} \sum_{j}\left(\left[V_{j} \rho, V_{j}\right]+\left[V_{j}, \rho V_{j}\right]\right),
$$

where $H$ denotes the open system's Hamiltonian and the operators $V_{k}$ and $V_{k}^{\dagger}$ defined on the Hilbert space of $H$, describe the interaction of open system with the environment. Given that, we have an interest in the set of Gaussian states, we introduce such quantum dynamical semi group that preserves this set. Consequently, $H$ is considered to be a polynomial of second degree in coordinates $Q_{1}$, $Q_{2}$ and momenta $P_{1}, P_{2}$ of the quantum oscillators. In this case, $V_{j}$ and $V_{j}^{\dagger}$ are taken as polynomials of first degree in these canonical observables. Then, in the linear space spanned by the coordinates and momenta, there exist only four linearly independent operators $V_{j}(j=1,2,3,4)$ [16] [44]. Moreover, the operators are non-Hermitian and describe the dissipation and decoherence due to interaction between the system and its environment. Using the transformations (2) and (6), the Lindblad operators in the NC phase-space, are given by [42] [43]:

$$
\left\{\begin{array}{l}
V_{j}=A_{j 1} P_{1}+A_{j 2} P_{2}+B_{j 1} Q_{1}+B_{j 2} Q_{2} \\
V_{j}^{\dagger}=A_{j 1}^{*} P_{1}+A_{j 2}^{*} P_{2}+B_{j 1}^{*} Q_{1}+B_{j 2}^{*} Q_{2}
\end{array}\right.
$$

with

$$
\left\{\begin{array}{l}
A_{j 1}=\frac{1}{\chi}\left(a_{j 1}+b_{j 2} \frac{\theta}{2 \hbar}\right) \cos (\Phi)+\frac{1}{\rho}\left(b_{j 2}+a_{j 1} \frac{\eta}{2 \hbar}\right) \sin (\Phi), \\
A_{j 2}=\frac{1}{\chi}\left(a_{j 1}-b_{j 1} \frac{\theta}{2 \hbar}\right) \cos (\Phi)+\frac{1}{\rho}\left(b_{j 1}-a_{j 2} \frac{\eta}{2 \hbar}\right) \sin (\Phi), \\
B_{j 1}=-\rho\left(a_{j 2}-b_{j 1} \frac{\theta}{2 \hbar}\right) \sin (\Phi)+\chi\left(b_{j 1}-a_{j 2} \frac{\eta}{2 \hbar}\right) \cos (\Phi), \\
B_{j 2}=-\rho\left(a_{j 1}+b_{j 2} \frac{\theta}{2 \hbar}\right) \sin (\Phi)+\chi\left(b_{j 2}+a_{j 1} \frac{\eta}{2 \hbar}\right) \cos (\Phi),
\end{array}\right.
$$


where $a_{j 1}, a_{j 2}, b_{j 1}, b_{j 2}$ are complex numbers. The constant term is omitted because its contribution to the generator $L$ is equivalent to the linear terms of the Hamiltonian in $P_{k}$ and $Q_{k}$. Then, the harmonic oscillator's Hamiltonian (8) can be rewritten in the form:

$$
H=H_{0}+c_{12} Q_{1} Q_{2}+\mu_{11} Q_{1} P_{1}+\mu_{22} Q_{2} P_{2}+d_{12} P_{1} P_{2},
$$

with

$$
H_{0}=\frac{1}{2 M_{1}} P_{1}^{2}+\frac{1}{2 M_{2}} P_{2}^{2}+\frac{M_{1}}{2} \Omega_{1}^{2} Q_{1}^{2}+\frac{M_{2}}{2} \Omega_{2}^{2} Q_{2}^{2} .
$$

Under the above conditions, Equation (13) becomes [5] [45]:

$$
\begin{aligned}
\frac{\mathrm{d} \rho}{\mathrm{d} t}= & -\frac{i}{\hbar}\left[H_{0}, \rho\right]-\frac{i}{2 \hbar}\left(\mu_{11}+\lambda_{11}\right)\left[Q_{1}, \rho P_{1}+P_{1} \rho\right] \\
& -\frac{i}{2 \hbar}\left(\mu_{22}+\lambda_{22}\right)\left[Q_{2}, \rho P_{2}+P_{2} \rho\right]-\frac{i}{2 \hbar} \lambda_{12}\left(\left[Q_{1}, \rho P_{2}+P_{2} \rho\right]\right. \\
& \left.-\left[P_{2}, \rho Q_{1}+Q_{1} \rho\right]\right)-\frac{i}{2 \hbar} \lambda_{21}\left(\left[Q_{2}, \rho P_{1}+P_{1} \rho\right]-\left[P_{1}, \rho Q_{2}+Q_{2} \rho\right]\right) \\
& -\frac{i}{2 \hbar}\left(d_{12}+\alpha_{12}\right)\left[P_{2}, \rho P_{1}+P_{1} \rho\right]-\frac{i}{2 \hbar}\left(c_{12}+\beta_{12}\right)\left[Q_{2}, \rho Q_{1}+Q_{1} \rho\right] \\
& +\frac{i}{2 \hbar}\left(\lambda_{11}-\mu_{11}\right)\left[P_{1}, \rho Q_{1}+Q_{1} \rho\right]+\frac{i}{2 \hbar}\left(\lambda_{22}-\mu_{22}\right)\left[P_{2}, \rho Q_{2}+Q_{2} \rho\right] \\
& +\frac{i}{2 \hbar}\left(\alpha_{12}-d_{12}\right)\left[P_{1}, \rho P_{2}+P_{2} \rho\right]+\frac{i}{2 \hbar}\left(\beta_{12}-c_{12}\right)\left[Q_{1}, \rho Q_{2}+Q_{2} \rho\right] \\
& -\frac{1}{\hbar^{2}} \sum_{k, l=1}^{2}\left[D_{P_{k} P_{l}}\left[Q_{k},\left[Q_{l}, \rho\right]\right]+D_{Q_{k} Q_{l}}\left[P_{k},\left[P_{l}, \rho\right]\right]\right. \\
& \left.-D_{Q_{k} P_{l}}\left(\left[Q_{k},\left[P_{l}, \rho\right]\right]+\left[P_{k},\left[Q_{l}, \rho\right]\right]\right)\right]
\end{aligned}
$$

where

$$
\begin{gathered}
D_{Q_{l} Q_{l}}=\frac{\hbar}{2} \sum_{j}\left|A_{j l}\right|^{2}, \quad D_{P_{l} P_{l}}=\frac{\hbar}{2} \sum_{j}\left|B_{j l}\right|^{2}, \quad D_{Q_{1} Q_{2}}=D_{Q_{2} Q_{1}}=\frac{\hbar}{2} \operatorname{Re}\left[\sum_{j} A_{j 1}^{*} A_{j 2}\right], \\
D_{P_{1} P_{2}}=D_{P_{2} P_{1}}=\frac{\hbar}{2} \operatorname{Re}\left[\sum_{j} B_{j 1}^{*} B_{j 2}\right], \quad D_{P_{l} Q_{l}}=D_{Q_{l} P_{l}}=-\frac{\hbar}{2} \operatorname{Re}\left[\sum_{j} A_{j l}^{*} B_{j l}\right], \\
D_{P_{1} Q_{2}}=D_{Q_{2} P_{1}}=-\frac{\hbar}{2} \operatorname{Re}\left[\sum_{j} A_{j 2}^{*} B_{j 1}\right], \quad D_{P_{2} Q_{1}}=D_{Q_{1} P_{2}}=-\frac{\hbar}{2} \operatorname{Re}\left[\sum_{j} A_{j 1}^{*} B_{j 2}\right], \\
\alpha_{12}=-\alpha_{21}=-\operatorname{Im}\left[\sum_{j} A_{j 1}^{*} A_{j 2}\right], \quad \beta_{12}=-\beta_{21}=-\operatorname{Im}\left[\sum_{j} B_{j 1}^{*} B_{j 2}\right], \\
\lambda_{11}=-\operatorname{Im}\left[\sum_{j} A_{j 1}^{*} B_{j 1}\right], \quad \lambda_{22}=-\operatorname{Im}\left[\sum_{j} A_{j 2}^{*} B_{j 2}\right], \\
\lambda_{12}=-\operatorname{Im}\left[\sum_{j} A_{j 1}^{*} B_{j 2}\right], \quad \lambda_{21}=-\operatorname{Im}\left[\sum_{j} A_{j 2}^{*} B_{j 1}\right] .
\end{gathered}
$$

The quantities $D_{P_{k} P_{l}}, D_{Q_{k} Q_{l}}, D_{P_{k} Q_{l}}$ and $D_{Q_{k} P_{l}}(k=l=1,2)$ are known as the diffusion coefficients, $\lambda_{k l}, \alpha_{12}, \alpha_{21}, \beta_{12}$ and $\beta_{21}$ the frictions constants. From the Cauchy-Schwarz inequality, the above coefficients satisfy the following fundamental constraints [2]: 
- The coefficients $D_{P_{l} P_{l}}, D_{Q_{l} Q_{l}}$, must be positive $\forall l$.

- $D_{P_{l} P_{l}} D_{Q_{l} Q_{l}}-D_{Q_{l} P_{l}}^{2}$ should be greater than the ratio $\frac{\lambda_{l l}^{2} \hbar^{2}}{4} \quad \forall l$.

- $D_{Q_{1} Q_{1}} D_{Q_{2} Q_{2}}-D_{Q_{1} Q_{2}}^{2}$ must be greater than $\frac{\alpha_{12}^{2} \hbar^{2}}{4}$, and

- $D_{P_{1} P_{1}} D_{P_{2} P_{2}}-D_{P_{1} P_{2}}^{2}$ must be greater than $\frac{\beta_{12}^{2} \hbar^{2}}{4}$.

In the particular case, we suppose that the asymptotic state of the considered open system is a Gibbs state $\left(\rho_{G}(\infty)=\frac{\mathrm{e}^{-\frac{H_{0}}{k T}}}{\operatorname{Tr}\left[\mathrm{e}^{-\frac{H_{0}}{k T}}\right]}\right)$, corresponding to a quantum harmonic oscillator in thermal equilibrium at temperature $T$ in two-dimensional phase-space. Under this assumption, the above quantum diffusion coefficients take the forms [45]:

$$
\left\{\begin{array}{l}
D_{P_{l} P_{l}}=\hbar M_{l} \Omega_{l} \frac{\lambda_{l l}+\mu_{l l}}{2} \operatorname{coth}\left(\frac{\hbar \Omega_{l}}{2 k T}\right) \\
D_{Q_{l} Q_{l}}=\frac{\lambda_{l l}-\mu_{l l}}{2} \frac{\hbar}{M_{l} \Omega_{l}} \operatorname{coth}\left(\frac{\hbar \Omega_{l}}{2 k T}\right)
\end{array}\right.
$$

other combinations being zero. In this case, the previous fundamental constraints are satisfied for example only if: $\lambda_{l l}>\mu_{l l}$ and $\left(\lambda_{l l}-\mu_{l l}\right) \operatorname{coth}\left(\frac{\hbar \Omega_{l}}{2 k T}\right) \geq \lambda_{l l}^{2}$, $\forall l=1,2$.

Let $\sigma_{A A}=\left\langle A^{2}\right\rangle-\langle A\rangle^{2}$ the dispersion (variance) of a given operator $A$, where $\langle A\rangle=\operatorname{Tr}[\rho A]$ is the expectation value of the operator $A, \rho$ the statistical operator (density matrix), and $\sigma_{A B}=\frac{\langle A B+B A\rangle}{2}-\langle A\rangle\langle B\rangle$ the correlation (covariance) of the operators $A$ and $B$. It was demonstrated in [46] [47] that, for any Hermitian operators $A$ and $B$, and for pure quantum states, the following generalized uncertainty relation holds:

$$
\sigma_{A A} \sigma_{B B}-\sigma_{A B}^{2} \geq \frac{1}{4}|\langle[A, B]\rangle|^{2} .
$$

From the master Equation (18), we obtain the following equation of motion for the expectation values of coordinates and momenta [22] [48]:

$$
\frac{\mathrm{d} n(t)}{\mathrm{d} t}=X n(t)
$$

where $n(t)=\left[\sigma_{Q_{1}}, \sigma_{Q_{2}}, \sigma_{P_{1}}, \sigma_{P_{2}}\right]^{\mathrm{T}}$ denotes the expectation vector and $X$ a $4 \times 4$ matrix defined by:

$$
X=\left(\begin{array}{cccc}
-\lambda_{11}+\mu_{11} & -\lambda_{21} & \frac{1}{M_{1}} & -\alpha_{12}+d_{12} \\
-\lambda_{12} & -\lambda_{22}+\mu_{22} & \alpha_{12}+d_{12} & \frac{1}{M_{2}} \\
-M_{1} \Omega_{1}^{2} & \beta_{12}-c_{12} & -\lambda_{11}-\mu_{11} & -\lambda_{12} \\
-\beta_{12}-c_{12} & -M_{2} \Omega_{2}^{2} & -\lambda_{21} & -\lambda_{22}-\mu_{22}
\end{array}\right)
$$


Solving Equation (21), we obtain

$$
n(t)=N(t) n(0)=\exp (X t) n(0),
$$

where $n(0)$ denotes the initial conditions. The matrix $n(t)$ must fulfill the condition

$$
\lim _{t \rightarrow \infty} n(t)=0 .
$$

However, for this limit to exist, $X$ must have only negative real eigenvalues parts. For this reason, we set $k_{12}=0$, such that $\mu_{l l}=d_{12}=c_{12}=0$, which lead the system to uncoupled oscillators. Under these conditions, the matrix $X$ becomes:

$$
X=\left(\begin{array}{cccc}
-\lambda_{11} & -\lambda_{21} & \frac{1}{M_{1}} & -\alpha_{12} \\
-\lambda_{12} & -\lambda_{22} & \alpha_{12} & \frac{1}{M_{2}} \\
-M_{1} \Omega_{1}^{2} & \beta_{12} & -\lambda_{11} & -\lambda_{12} \\
-\beta_{12} & -M_{2} \Omega_{2}^{2} & -\lambda_{21} & -\lambda_{22}
\end{array}\right)
$$

In order to evaluate the exponential matrix $N(t)$, it is important to first diagonalize the matrix $X$ by solving the corresponding secular equation i.e.

$$
\operatorname{det}(X-z \mathbb{I})=0,
$$

where $z$ is the eigenvalues of $X$ and $\mathbb{I}$ the unit matrix. From Equation (26), one obtains an equation of fourth order with respect to the eigenvalues $Z$, which can be easily solved. Let us for simplicity consider the particular case where $\beta_{12}=\alpha_{12}=\lambda_{12}=\lambda_{21}=0$, then the secular equation obtained is:

$$
\left[\left(\lambda_{11}+z\right)^{2}+\Omega_{1}^{2}\right]\left[\left(\lambda_{22}+z\right)^{2}+\Omega_{2}^{2}\right]=0 .
$$

The resolution of this equation provides $z_{1}=-\lambda_{+}+i \Omega_{+}, z_{2}=-\lambda_{-}+i \Omega_{-}$, $z_{3}=-\lambda_{+}-i \Omega_{+}$and $z_{4}=-\lambda_{-}-i \Omega_{-}$, where $\lambda_{+}=\lambda_{11}, \lambda_{-}=\lambda_{22}, \Omega_{+}=\Omega_{1}$, and $\Omega_{-}=\Omega_{2}$. To fulfill the condition of Equation (24), only the positive values of $\lambda_{+}$and $\lambda_{-}$are considered. Thus, using the eigenvalues $z_{n}$ of $X$, the time-dependent matrix $N(t)$ can be obtained as follows:

$$
N_{i j}(t)=\sum_{n} M_{i n} \exp \left(z_{n} t\right) M_{n j}^{-1},
$$

where the matrix $M$ constitutes the eigenvectors of $X$ :

$$
\sum_{j} X_{i j} M_{j n}=z_{n} M_{i n} \text {. }
$$

Under the conditions $N_{i j}(t=0)=\delta_{i j}$ and $\mathrm{d} N_{i j}(t) /\left.\mathrm{d} t\right|_{t=0}=X_{i j}$. The expressions of the matrix elements $N_{i j}$, can subsequently be obtained as:

$$
N(t)=\left(\begin{array}{cccc}
\mathrm{e}^{-\lambda_{11} t} \cos \Omega_{1} t & 0 & \frac{1}{M_{1} \Omega_{1}} \mathrm{e}^{-\lambda_{11} t} \sin \Omega_{1} t & 0 \\
0 & \mathrm{e}^{-\lambda_{22} t} \cos \Omega_{2} t & 0 & \frac{1}{M_{2} \Omega_{2}} \mathrm{e}^{-\lambda_{22} t} \sin \Omega_{2} t \\
-M_{1} \Omega_{1} \mathrm{e}^{-\lambda_{11} t} \sin \Omega_{1} t & 0 & \mathrm{e}^{-\lambda_{11} t} \cos \Omega_{1} t & 0 \\
0 & -M_{2} \Omega_{2} \mathrm{e}^{-\lambda_{22} t} \sin \Omega_{2} t & 0 & \mathrm{e}^{-\lambda_{22} t} \cos \Omega_{2} t
\end{array}\right) .
$$


Considering Equation (23) and the initial conditions defined above, one get:

$$
\left\{\begin{array}{l}
\sigma_{Q_{k}}(t)=\mathrm{e}^{-\lambda_{k k} t}\left[\sigma_{Q_{k}}(0) \cos \left(\Omega_{k} t\right)+\frac{1}{M_{k} \Omega_{k}} \sigma_{P_{k}}(0) \sin \left(\Omega_{k} t\right)\right], k=1,2 . \\
\sigma_{P_{k}}(t)=\mathrm{e}^{-\lambda_{k k} t}\left[-M_{k} \Omega_{k} \sigma_{Q_{k}}(0) \sin \left(\Omega_{k} t\right)+\sigma_{P_{k}}(0) \cos \left(\Omega_{k} t\right)\right],
\end{array}\right.
$$

Thus, we can observe that, the expectation values of the coordinates and momenta decay quickly due to the exponential factors $\mathrm{e}^{-\lambda_{11} t}$ and $\mathrm{e}^{-\lambda_{22} t}$ and vanish when $t \rightarrow \infty$. Let us now determine the variance and covariance matrices. For this reason, let $\sigma(t)$ and $D$ be respectively the covariance and the diffusion coefficients matrices where the elements are defined as follows:

$$
D=\left(\begin{array}{cccc}
D_{Q_{1} Q_{1}} & D_{Q_{1} Q_{2}} & D_{Q_{1} P_{1}} & D_{Q_{1} P_{2}} \\
D_{Q_{2} Q_{1}} & D_{Q_{2} Q_{2}} & D_{Q_{2} P_{1}} & D_{Q_{2} P_{2}} \\
D_{P_{1} Q_{1}} & D_{P_{1} Q_{2}} & D_{P_{1} P_{1}} & D_{P_{1} P_{2}} \\
D_{P_{2} Q_{1}} & D_{P_{2} Q_{2}} & D_{P_{2} P_{1}} & D_{P_{2} P_{2}}
\end{array}\right) \text { and } \sigma(t)=\left(\begin{array}{cccc}
\sigma_{Q_{1} Q_{1}} & \sigma_{Q_{1} Q_{2}} & \sigma_{Q_{1} P_{1}} & \sigma_{Q_{1} P_{2}} \\
\sigma_{Q_{2} Q_{1}} & \sigma_{Q_{2} Q_{2}} & \sigma_{Q_{2} P_{1}} & \sigma_{Q_{2} P_{2}} \\
\sigma_{P_{1} Q_{1}} & \sigma_{P_{1} Q_{2}} & \sigma_{P_{1} P_{1}} & \sigma_{P_{1} P_{2}} \\
\sigma_{P_{2} Q_{1}} & \sigma_{P_{2} Q_{2}} & \sigma_{P_{2} P_{1}} & \sigma_{P_{2} P_{2}}
\end{array}\right) \text {, }
$$

Thus, from the master Equation (18), the equations of motion corresponding to the quantum correlations of canonical observables $Q_{1}, Q_{2}$ and $P_{1}, P_{2}$ are the following [48] [49]:

$$
\frac{\mathrm{d} \sigma}{\mathrm{d} t}=X \sigma+\sigma X^{\mathrm{T}}+2 D
$$

where the matrix $X$ is defined in Equation (25), with $X^{\mathrm{T}}$ its transposed matrix. The time-dependent solution of (33) is given by:

$$
\sigma(t)=N(t)(\sigma(0)-\sigma(\infty)) N^{\mathrm{T}}(t)+\sigma(\infty),
$$

where $N(t)$ is defined in Equation (30). The matrix $\sigma(\infty)$ is time independent and solves the static problem Equation (33) (i.e. $\frac{\mathrm{d} \sigma}{\mathrm{d} t}=0$ ). Assuming that the limit of $\sigma(t)$ exists as $t$ goes to infinity (i.e. $\lim _{t \rightarrow \infty} \sigma(t)$ exists), then one has $\sigma(\infty)=\lim _{t \rightarrow \infty} \sigma(t)$. We can therefore, obtain the solutions of Equation (33) giving the elements of $\sigma(t)$. For illustration, the elements $\sigma_{Q_{k} Q_{k}}, \sigma_{P_{k} P_{k}}$ and $\sigma_{P_{k} Q_{k}}$ $(k=1,2)$ are given by:

$$
\left\{\begin{aligned}
\sigma_{Q_{k} Q_{k}}(t)= & \mathrm{e}^{-2 \lambda_{k k} t}\left(\cos ^{2}\left(\Omega_{k} t\right)\left(\sigma_{Q_{k} Q_{k}}(0)-\sigma_{Q_{k} Q_{k}}(\infty)\right)+\frac{1}{M_{k} \Omega_{k}} \sin \left(2 \Omega_{k} t\right)\left(\sigma_{Q_{k} P_{k}}(0)-\sigma_{Q_{k} P_{k}}(\infty)\right)\right. \\
& \left.+\frac{1}{M_{k}^{2} \Omega_{k}^{2}}\left(\sigma_{P_{k} P_{k}}(0)-\sigma_{P_{k} P_{k}}(\infty)\right) \sin ^{2}\left(\Omega_{k} t\right)\right)+\sigma_{Q_{k} Q_{k}}(\infty) \\
\sigma_{P_{k} P_{k}}(t)= & \mathrm{e}^{-2 \lambda_{k k} t}\left(M_{k}^{2} \Omega_{k}^{2} \sin ^{2}\left(\Omega_{k} t\right)\left(\sigma_{Q_{k} Q_{k}}(0)-\sigma_{Q_{k} Q_{k}}(\infty)\right)-M_{k} \Omega_{k} \sin \left(2 \Omega_{k} t\right)\left(\sigma_{q_{k} P_{k}}(0)-\sigma_{q_{k} P_{k}}(\infty)\right)\right. \\
& \left.+\left(\sigma_{P_{k} P_{k}}(0)-\sigma_{P_{k} P_{k}}(\infty)\right) \cos ^{2}\left(\Omega_{k} t\right)\right)+\sigma_{P_{k} P_{k}}(\infty) ; \\
\sigma_{P_{k} Q_{k}}(t)= & \mathrm{e}^{-2 \lambda_{k k} t}\left(-\frac{M_{k} \Omega_{k}}{2} \sin \left(2 \Omega_{k} t\right)\left(\sigma_{Q_{k} Q_{k}}(0)-\sigma_{Q_{k} Q_{k}}(\infty)\right)+\cos \left(2 \Omega_{k} t\right)\left(\sigma_{P_{k} Q_{k}}(0)-\sigma_{P_{k} Q_{k}}(\infty)\right)\right. \\
& \left.+\frac{1}{2 M_{k} \Omega_{k}}\left(\sigma_{P_{k} P_{k}}(0)-\sigma_{P_{k} P_{k}}(\infty)\right) \sin \left(2 \Omega_{k} t\right)\right)+\sigma_{P_{k} Q_{k}}(\infty)
\end{aligned}\right.
$$


where the remaining elements can be easily found following the same approach. It can be observed that the matrix elements $\sigma_{i j}$ decay rapidly to zero due to the exponential terms $\exp \left(-2 \lambda_{11} t\right), \exp \left(-2 \lambda_{22} t\right)$ and $\exp \left(-\left(\lambda_{11}+\lambda_{22}\right) t\right)$. The matrix elements of $\sigma(\infty)$ depend on $X$ and $D$, thus should be evaluated using the relation [22]:

$$
\sigma(\infty)=2 \int_{0}^{\infty} N\left(t^{\prime}\right) D N\left(t^{\prime}\right)^{\mathrm{T}} \mathrm{d} t^{\prime}
$$

This relation is remarkably important, since it gives a very simple connection between the asymptotic values $(t \rightarrow \infty)$ of $\sigma(t)$ and the diffusion coefficients $D$. One observe that the asymptotic values $\sigma_{Q_{k} Q_{k}}(\infty), \sigma_{P_{k} P_{k}}(\infty)$ and $\sigma_{P_{k} Q_{l}}(\infty)$ do not depend on initial values $\sigma_{Q_{k} Q_{k}}(0), \sigma_{P_{k} P_{k}}(0)$ and $\sigma_{P_{k} Q_{l}}(0)$. The initial state represents a correlated coherent state with variance and covariance of coordinates and momenta defined as [15]:

$$
\sigma_{Q_{k} Q_{k}}(0)=\frac{\hbar \delta}{2 M_{k} \Omega_{k}}, \sigma_{P_{k} P_{k}}(0)=\frac{\hbar M_{k} \Omega_{k}}{2 \delta\left(1-r_{k}^{2}\right)} \text { and } \sigma_{P_{k} Q_{k}}(0)=\frac{\hbar r_{k}}{2 \delta \sqrt{1-r_{k}^{2}}} .
$$

Here, $\delta$ represents the squeezing parameter and $r_{k}$ the correlation coefficient at $t=0$ with $\left|r_{k}\right|<1$, where $r_{k}=\frac{\sigma_{P_{k} Q_{k}}}{\sqrt{\sigma_{Q_{k} Q_{k}} \sigma_{P_{k} P_{k}}}}$. In the case of a thermal bath where $\mu_{l l}=0$, with the coefficients (19) one can deduce that

$$
\begin{aligned}
& \sigma_{Q_{k} Q_{k}}(\infty)=\frac{\hbar}{2 M_{k} \Omega_{k}} \operatorname{coth}\left(\frac{\hbar \Omega_{k}}{2 k T}\right), \\
& \sigma_{P_{k} P_{k}}(\infty)=\frac{M_{k} \Omega_{k} \hbar}{2} \operatorname{coth}\left(\frac{\hbar \Omega_{k}}{2 k T}\right) \text { and } \sigma_{P_{k} Q_{l}}(\infty)=0 .
\end{aligned}
$$

The semigroup dynamics density operator which must hold for a quantum Markovian process is valid only for the weak-coupling regime, with the damping $\lambda_{k k}$ obeying the inequality $\lambda_{k k} \ll \Omega_{k}$. Moreover, the diffusion coefficients $D_{Q_{1} Q_{2}}, D_{P_{2} Q_{1}}, D_{P_{1} Q_{2}}$ and $D_{P_{1} P_{2}}$ are generally zero for uncoupled oscillators interacting with an usual environment. Consequently, the expectation values $\sigma_{Q_{1} Q_{2}}, \sigma_{P_{2} Q_{1}}, \sigma_{P_{1} Q_{2}}$ and $\sigma_{P_{1} P_{2}}$ vanish when $t \rightarrow \infty$. It is a very interesting point that the general theory of Lindblad allows coupling via the environment between uncoupled oscillators $\left(d_{12}=0, c_{12}=0, \mu_{l l}=0,(l=1 ; 2)\right)$. Furthermore, according to the definition of the parameters in terms of the vectors $A_{j k}$ and $B_{j k}$, the diffusion coefficients above can be different from zero. In this case, a structure of the environment is reflected in the motion of the oscillators.

\section{Density Matrix and Wigner Distribution Function}

In this section, the NC anisotropic oscillator interacting with the usual environment is considered in the same idea presented in the previous section, but with $k_{12}=0, \alpha_{12}=0, \beta_{12}=0, \lambda_{12}=0, \lambda_{21}=0$. Considering these conditions and following the method developed by Lampo et al. [6] in the one dimensional case, we derive the evolution in coordinate representation from Equation (18) as: 


$$
\begin{aligned}
\frac{\mathrm{d} \rho}{\mathrm{d} t}= & \sum_{k=1}^{2}\left\{\left[\frac{i \hbar}{M_{k}}\left(\frac{\partial^{2}}{\partial Q_{k}^{2}}-\frac{\partial^{2}}{\partial Q_{k}^{\prime 2}}\right)-\frac{i M_{k} \Omega_{k}^{2}}{2 \hbar}\left(Q_{k}^{2}-Q_{k}^{\prime 2}\right)\right] \rho\right. \\
& \left.-\frac{\lambda_{k k}}{2}\left(Q_{k}-Q_{k}^{\prime}\right)\left(\frac{\partial}{\partial Q_{k}}-\frac{\partial}{\partial Q_{k}^{\prime}}\right) \rho\right\} \\
& +\sum_{k=1}^{2}\left[\frac{\lambda_{k k}}{2}\left[\left(Q_{k}+Q_{k}^{\prime}\right)\left(\frac{\partial}{\partial Q_{k}}+\frac{\partial}{\partial Q_{k}^{\prime}}\right)+2\right] \rho\right. \\
& \left.+D_{Q_{k} Q_{k}}\left(\frac{\partial}{\partial Q_{k}}+\frac{\partial}{\partial Q_{l}^{\prime}}\right)^{2} \rho-\frac{D_{P_{k} P_{k}}}{\hbar^{2}}\left(Q_{k}-Q_{k}^{\prime}\right)^{2} \rho\right] \\
& +\sum_{k \neq l=1}^{2}\left[-\frac{i D_{Q_{k} P_{l}}}{\hbar}\left(Q_{k}-Q_{k}^{\prime}\right)\left(\frac{\partial}{\partial Q_{l}}+\frac{\partial}{\partial Q_{l}^{\prime}}\right) \rho\right. \\
& \left.+D_{Q_{k} Q_{l}}\left(\frac{\partial}{\partial Q_{k}}+\frac{\partial}{\partial Q_{k}^{\prime}}\right)\left(\frac{\partial}{\partial Q_{l}}+\frac{\partial}{\partial Q_{l}^{\prime}}\right) \rho\right] \\
& -\sum_{k \neq l=1}^{2} \frac{D_{P_{k} P_{l}}}{\hbar^{2}}\left(Q_{k}-Q_{k}^{\prime}\right)\left(Q_{l}-Q_{l}^{\prime}\right) \rho \\
& -\sum_{k=1}^{2} \frac{2 i D_{Q_{k} P_{k}}}{\hbar}\left(Q_{k}-Q_{k}^{\prime}\right)\left(\frac{\partial}{\partial Q_{k}}+\frac{\partial}{\partial Q_{k}^{\prime}}\right) \rho
\end{aligned}
$$

Following [21], we can also transform the master Equation (18) for the density operator into the following Fokker-Planck-type equation satisfied by the Wigner distribution function $W\left(Q_{1}, Q_{2}, P_{1}, P_{2}, t\right)$ :

$$
\begin{aligned}
\frac{\partial W}{\partial t}= & \sum_{k=1}^{2}\left\{\left[-\frac{P_{k}}{M_{k}} \frac{\partial W}{\partial Q_{k}}+M_{k} \Omega_{k}^{2} \frac{\partial W}{\partial P_{k}}\right]+\lambda_{k k}\left(\frac{\partial\left(P_{k} W\right)}{\partial P_{k}}+\frac{\partial\left(Q_{k} W\right)}{\partial Q_{k}}\right)\right\} \\
& +\sum_{k=1}^{2}\left[D_{Q_{k} Q_{k}} \frac{\partial^{2} W}{\partial^{2} Q_{k}}+D_{P_{k} P_{k}} \frac{\partial^{2} W}{\partial^{2} P_{k}}+2 D_{Q_{k} P_{k}} \frac{\partial^{2} W}{\partial Q_{k} \partial P_{k}}\right] \\
& +\sum_{k \neq l=1}^{2}\left[2 D_{Q_{k} Q_{l}} \frac{\partial^{2} W}{\partial Q_{k} \partial Q_{l}}+2 D_{P_{k} P_{l}} \frac{\partial^{2} W}{\partial P_{k} \partial P_{l}}+2 D_{Q_{k} P_{l}} \frac{\partial^{2} W}{\partial Q_{k} \partial P_{l}}\right] .
\end{aligned}
$$

In Equation (40), the first four terms on the right-hand side give a purely unitary evolutions, the second four terms are the dissipative terms and have a damping effect (exchange of energy with environment), the last twelve terms are diffusive (noise) terms and produce fluctuation effects in the evaluation of the system. Moreover $D_{P_{k} P_{k}}$ promotes diffusion in momentum and generates decoherence in coordinate $Q_{k}$. It reduces the off-diagonal terms, responsible for correlations between spatially separated pieces of the wave packet. Analogically, $D_{Q_{k} Q_{k}}$ promotes diffusion in coordinate and generates decoherence in momentum $P_{k}$. The terms $D_{Q_{k} Q_{l}}, D_{Q_{k} P_{l}}, D_{Q_{k} P_{k}}$, and $D_{P_{k} P_{l}}(k \neq l=1,2)$ are the so-called "anomalous diffusion" terms. They promote diffusion in the variables $P_{k} P_{l}+P_{l} P_{k}, Q_{k} P_{l}+P_{l} Q_{k}, Q_{k} P_{k}+P_{k} Q_{k}$, and $Q_{k} Q_{l}+Q_{l} Q_{k}$ respectively. Just like the other diffusion terms but they do not generate decoherence [5]. To solve Equation (40), we have to first recall that the diffusion coefficients $D_{Q_{1} Q_{2}}, D_{Q_{1} P_{2}}$, $D_{Q_{2} P_{1}}, D_{P_{1} P_{2}}$ are well known and are generally zero for uncoupled oscillators. In addition, introducing the matrix notation $\left\langle Q_{k} Q_{l}\right\rangle=G_{k l},\left\langle P_{k} P_{l}\right\rangle=E_{k l}$ and 
$\left\langle Q_{k} P_{l}\right\rangle=F_{l k}$, which are the coefficients of the matrices $G, E$ and $F$ respectively, then for an initial Gaussian, the density matrix solution of Equation 40 ) and the Wigner function solution of Equation (40) are given by [21] [50] [51] [52]:

$$
\begin{aligned}
& \rho\left(Q_{1}, Q_{2}, Q_{1}^{\prime}, Q_{2}^{\prime}, t\right) \\
& =\frac{1}{2 \pi \sqrt{\operatorname{det} G}} \exp \left\{-\frac{1}{2}\left\{\left((R-\bar{Q})^{\mathrm{T}}, r^{\mathrm{T}}\right)\left[\begin{array}{cc}
G^{-1} & -\frac{i}{\hbar} H \\
-\frac{i}{\hbar} H^{\mathrm{T}} & \frac{\beta}{\hbar^{2}}
\end{array}\right]\left(\begin{array}{c}
R-\bar{Q}) \\
r
\end{array}\right)\right\}+\frac{i}{\hbar} \sum_{k=1}^{2} \sigma_{P_{k}} r_{k}\right\},
\end{aligned}
$$

and

$$
W\left(Q_{1}, Q_{2}, P_{1}, P_{2}, t\right)=\frac{1}{\sqrt{\operatorname{det}(2 \pi \sigma(t))}} \exp \left[-\frac{1}{2}(y-n(t)) \sigma(t)^{-1}(y-n(t))^{\mathrm{T}}\right],
$$

where $R=\left(\begin{array}{c}\frac{Q_{1}+Q_{1}^{\prime}}{2} \\ \frac{Q_{2}+Q_{2}^{\prime}}{2}\end{array}\right), \quad \bar{Q}=\left(\begin{array}{c}\sigma_{Q_{1}} \\ \sigma_{Q_{2}}\end{array}\right), r=\left(\begin{array}{c}Q_{1}-Q_{1}^{\prime} \\ Q_{2}-Q_{2}^{\prime}\end{array}\right), H=G^{-1} F^{\mathrm{T}}$ and $\beta=E-F G^{-1} F^{\mathrm{T}}$, with $E=\left[\begin{array}{cc}\sigma_{P_{1} P_{1}} & 0 \\ 0 & \sigma_{P_{2} P_{2}}\end{array}\right], \quad F=\left[\begin{array}{cc}\sigma_{Q_{1} P_{1}} & 0 \\ 0 & \sigma_{Q_{2} P_{2}}\end{array}\right]$, and $G=\left[\begin{array}{cc}\sigma_{Q_{1} Q_{1}} & 0 \\ 0 & \sigma_{Q_{2} Q_{2}}\end{array}\right], y=\left(Q_{1}, Q_{2}, P_{1}, P_{2}\right) . n(t)=\left(\sigma_{Q_{1}}, \sigma_{Q_{2}}, \sigma_{P_{1}}, \sigma_{P_{2}}\right)$ denotes the expectation vector, and $\sigma(t)$ is the dispersion (correlation) matrix defined by:

$$
\sigma(t)=\left(\begin{array}{ll}
G & F \\
F & E
\end{array}\right) .
$$

Thus, in the case of a thermal bath and when the asymptotic state is a Gibbs state and considering Equation (38), we obtain the following steady state solution:

$$
\begin{aligned}
& \left\langle Q_{1} Q_{2}|\rho(\infty)| Q_{1}^{\prime} Q_{2}^{\prime}\right\rangle=\rho_{1} \rho_{2} \\
& =\left[\sqrt{\frac{M_{1} \Omega_{1}}{\pi \hbar \operatorname{coth} \varepsilon_{1}}} \exp \left\{\frac{-M_{1} \Omega_{1}}{4 \hbar}\left[\frac{\left(Q_{1}+Q_{1}^{\prime}\right)^{2}}{\operatorname{coth} \varepsilon_{1}}+\operatorname{coth} \varepsilon_{1}\left(Q_{1}-Q_{1}^{\prime}\right)^{2}\right]\right\}\right] \\
& \times\left[\sqrt{\frac{M_{2} \Omega_{2}}{\pi \hbar \operatorname{coth} \varepsilon_{2}}} \exp \left\{\frac{-M_{2} \Omega_{2}}{4 \hbar}\left[\frac{\left(Q_{2}+Q_{2}^{\prime}\right)^{2}}{\operatorname{coth} \varepsilon_{2}}+\operatorname{coth} \varepsilon_{2}\left(Q_{2}-Q_{2}^{\prime}\right)^{2}\right]\right\}\right]
\end{aligned}
$$

and

$$
\begin{aligned}
& W_{\infty}=W_{1} W_{2} \\
& =\left[\frac{1}{\pi \hbar \operatorname{coth} \varepsilon_{1}} \exp \left\{-\frac{1}{\hbar \operatorname{coth} \varepsilon_{1}}\left[M_{1} \Omega_{1} Q_{1}^{2}+\frac{1}{M_{1} \Omega_{1}} P_{1}^{2}\right]\right\}\right] \\
& \times\left[\frac{1}{\pi \hbar \operatorname{coth} \varepsilon_{2}} \exp \left\{-\frac{1}{\hbar \operatorname{coth} \varepsilon_{2}}\left[M_{2} \Omega_{2} Q_{2}^{2}+\frac{1}{M_{2} \Omega_{2}} P_{2}^{2}\right]\right\},\right.
\end{aligned}
$$

with $\varepsilon_{k}=\frac{\hbar \Omega_{k}}{k T}$. We observe that, all stationary solutions to the evolution equa- 
tions obtained in the long time limit are possible as a result of a balance between the wave packet spreading induced by the Hamiltonian and the localizing effect of the Lindblad operators. Figure 1 and Figure 2 depict the behavior of the density matrix and the Wigner distribution functions respectively. It can be observed that both exhibit a Gaussian behavior with the amplitudes which strongly depend on the NC parameters. The diagonal elements of the density matrix (i.e. $\left.Q_{k}=Q_{k}^{p}, k=1,2\right)$ represent the probability of finding the system in this position, while the off-diagonal elements introduce the correlation in the density matrix between the points $Q_{k}$ and $Q_{k}^{p}, k=1,2$.

\section{Results and Discussion}

In this section, we mainly focus on the decoherence time scale and the total energy of a two-dimensional harmonic oscillator in an NC phase-space. For the reasons of simplicity, let $\theta=\alpha \times 10^{-38} \mathrm{~m}^{2}$ and $\eta=\beta \times 10^{-60} \mathrm{~kg}^{2} \cdot \mathrm{m}^{2} \cdot \mathrm{s}^{-2}$, where $\theta$ and $\eta$ are the NC parameters, whose values are selected following the works of refs. [38] [39]. It is worth noting that $\alpha$ and $\beta$ are dimensionless.

\subsection{Decoherence Time Scale}

As previously mentioned, the diffusion coefficients in momentum $D_{P_{k} P_{k}}$ produces decoherence along coordinates $Q_{k}$. Mathematically, classical limit is often associated with the size of Planck's constant. In the macroscopic limit, this

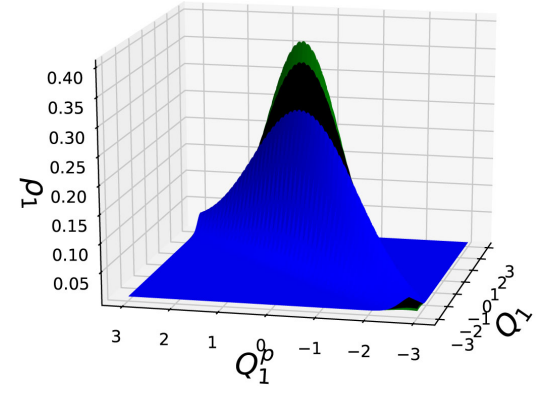

(a)

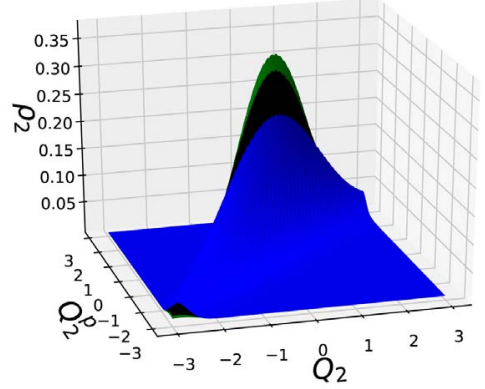

(b)

Figure 1. Density matrix $\rho_{\infty}$ with respect to the coordinates in the $Q_{1}$-direction (Figure 1 (a)) and in the $Q_{2}$-direction (Figure $1(\mathrm{~b})$ ) respectively.

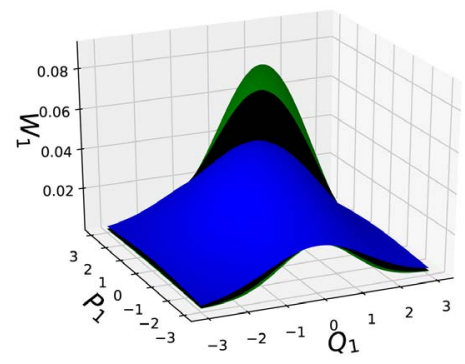

(a)

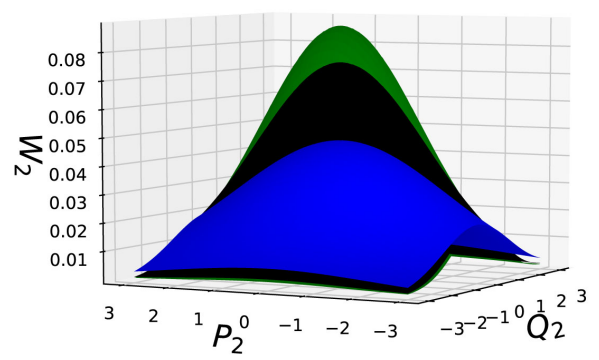

(b)

Figure 2. Wigner function $W_{\infty}$ with respect to both the coordinates and momentum in the $Q_{1}$-direction (Figure 2(a)) and in the $Q_{2}$-direction (Figure 2(b)) respectively. 
Planck constant $\hbar$ is negligible compared to other quantities with dimension of action, such as $\sqrt{D_{P_{1} P_{1}}\left\langle\left(Q_{1}-Q_{1}^{\prime}\right)^{2}\right\rangle+D_{P_{2} P_{2}}\left\langle\left(Q_{2}-Q_{2}^{\prime}\right)^{2}\right\rangle}$. Thus, in this condition, the terms of Equation (39) containing the quantity $-\frac{D_{P_{k} P_{k}}}{\hbar^{2}}$ dominate on others.

To kindly appreciate its effects, let us rewrite an explicit solution of the master Equation (Equation (39)) approximating the right hand side by the dominating terms. In that case, the following time depend evolution of the density matrix is obtained:

$$
\begin{aligned}
& \left\langle Q_{1} Q_{2}|\rho(t)| Q_{1}^{\prime} Q_{2}^{\prime}\right\rangle \\
& =\left\langle Q_{1} Q_{2}|\rho(0)| Q_{1}^{\prime} Q_{2}^{\prime}\right\rangle \exp \left\{-\left[\frac{D_{P_{1} P_{1}}}{\hbar^{2}}\left(Q_{1}-Q_{1}^{\prime}\right)^{2}+\frac{D_{P_{2} P_{2}}}{\hbar^{2}}\left(Q_{2}-Q_{2}^{\prime}\right)^{2}\right] t\right\} \\
& =\left\langle Q_{1} Q_{2}|\rho(0)| Q_{1}^{\prime} Q_{2}^{\prime}\right\rangle \exp \left\{-\frac{1}{t_{\text {deco }}} t\right\} .
\end{aligned}
$$

We can thus, easily deduce the decoherence time scale as:

$$
t_{\text {deco }}=\frac{\hbar^{2}}{D_{P_{1} P_{1}}\left(Q_{1}-Q_{1}^{\prime}\right)^{2}+D_{P_{2} P_{2}}\left(Q_{2}-Q_{2}^{\prime}\right)^{2}},
$$

which reduces to:

$$
t_{\text {deco }}=\frac{2 \hbar}{\lambda_{11} M_{1} \Omega_{1} \sigma_{Q_{1} Q_{1}}(0) \operatorname{coth} \varepsilon_{1}+\lambda_{22} M_{2} \Omega_{2} \sigma_{Q_{2} Q_{2}}(0) \operatorname{coth} \varepsilon_{2}},
$$

where the quantities $\left(Q_{k}-Q_{k}^{\prime}\right)^{2}$ are in order of the initial dispersion in coordinates $\sigma_{Q_{k} Q_{k}}(0)$, in the particular case of thermal bath (i.e. at zero temperature). Considering Equation (37), Equation (48) becomes:

$$
t_{\text {deco }}=\frac{4}{\lambda_{11} \delta \operatorname{coth} \varepsilon_{1}+\lambda_{22} \delta \operatorname{coth} \varepsilon_{2}},
$$

with $\tau_{1}=\frac{2 k T}{\hbar \Omega_{1}}=\frac{1}{\varepsilon_{1}}$ and $\tau_{2}=\frac{2 k T}{\hbar \Omega_{2}}=\frac{1}{\varepsilon_{2}}$. The quantity $t_{\text {deco }}$ defines the so-called decoherence time scale which as we previously mentioned defines the particular time that the system initially quantum, starts behaving classically (i.e. the particular time that the system starts losing its quantum properties). Based on the above analytical expressions, we have numerically simulated the decoherence time scale as function of the temperature on one hand and as function of the cutting frequency (magnetic field) on the other hand. One can easily observe on Figure 3(a) that, the NCty effects present significant impacts on this time scale, and thus, considerably affect decoherence in the system in the sense that, the decoherence time scale increases as the NC parameters increase. However, this time scale decreases asymptotically in the case of NC phase-space as well as in the commutative phase-space case, and similar effects are also observed as in the case of total energy since the NCty effects are not more observable for high temperature. It is important to mention that, the NCty effects contribution to decoherence for zero temperature coincides with that of the commutative space since 
the system receives very small feedback from the environment at this temperature. We can conclude that, even if the NCty effects increase decoherence in the system at high temperature, the system losses its coherence properties and becomes decoherent, because the decoherence time goes to zero for high temperature. Moreover, it is observed in Figure $3(\mathrm{~b})$ that, the decoherence time scale increases as the magnetic field increases, in the case of NC phase-space as well as in the case of commutative phase-space, but very slowly in the latest case. Under lower magnetic field effects, the decoherence time scale is different from zero implying that even at this temperature, the system still receives feedback from its environment. In addition, the decoherence time scale increases asymptotically to a fixed value in the case of NC phase-space as well as in the case of commutative phase-space. This constant value defines the time during which the system loses its coherence properties and becomes decoherent (the system exhibits classical behaviour). The above results are confirmed by Figure 4, plotting the decoherence time scale versus simultaneously the NC parameters in the $\mathrm{x}$ - and $y$-directions, where we can easily observe that it increases with both parameters.

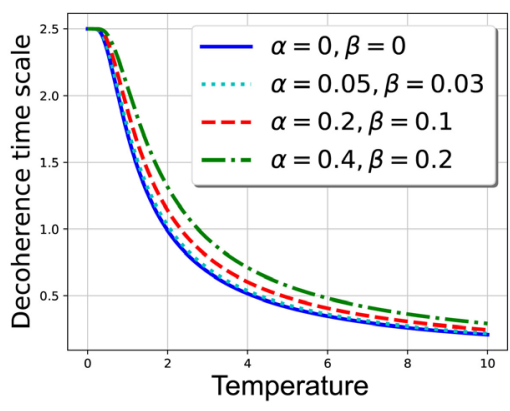

(a)

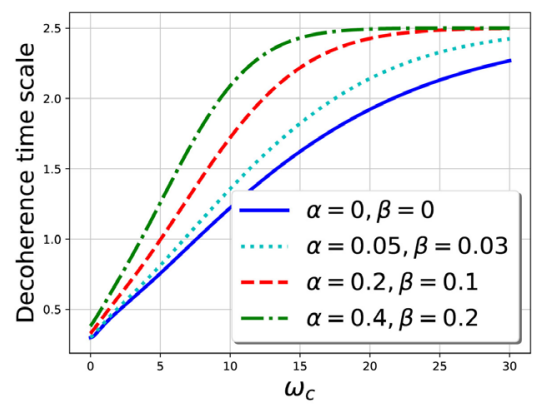

(b)

Figure 3. Decoherence time scale of an harmonic oscillator in two-dimensional phase-space with respect to the cutting frequency $\omega_{c}$ and with respect to the temperature $T$ both as function of the NC phase-space parameters.

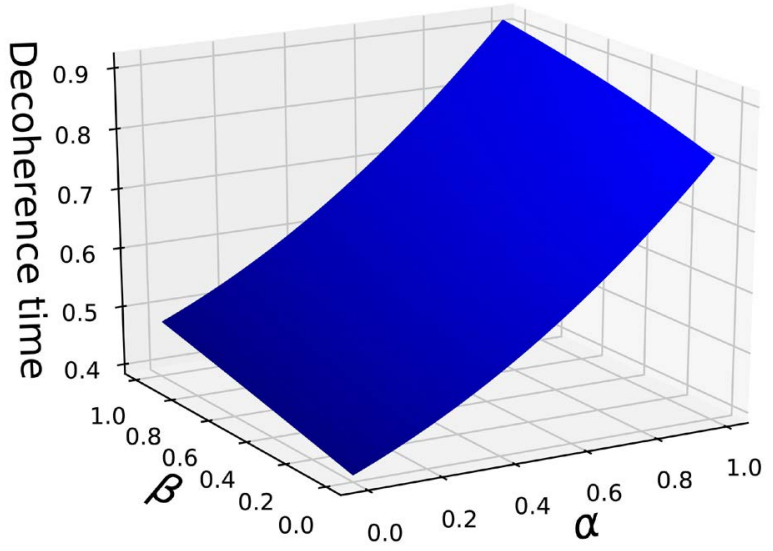

Figure 4. Decoherence time scale of an harmonic oscillator in two-dimensional phase-space with respect to both the NC parameters in the $\mathrm{x}$ - and $\mathrm{y}$-directions, for a fixed temperature $T=5 \mathrm{~K}$ and a fixed magnetic field $\omega_{c}=1.5$. 


\subsection{Total Energy of the System}

The total energy of the anisotropic oscillator in a NC phase space can be derived from the mean value of the Hamiltonian as:

$$
\begin{aligned}
\langle H\rangle= & \frac{\sigma_{P_{1} P_{1}}}{2 M_{1}}(t)+\frac{\sigma_{P_{2} P_{2}}}{2 M_{2}}(t)+\frac{1}{2} M_{1} \Omega_{1}^{2} \sigma_{Q_{1} Q_{1}}(t)+\frac{1}{2} M_{2} \Omega_{2}^{2} \sigma_{Q_{2} Q_{2}}(t) \\
& +\mu_{11} \sigma_{P_{1} Q_{1}}(t)+\mu_{22} \sigma_{P_{2} Q_{2}}(t)+d_{12} \sigma_{P_{1} P_{2}}(t)+c_{12} \sigma_{Q_{1} Q_{2}}(t) \\
& +\frac{1}{2 M_{1}} \sigma_{P_{1}}^{2}(t)+\frac{1}{2 M_{2}} \sigma_{P_{2}}^{2}(t)+\frac{1}{2} M_{1} \Omega_{1}^{2} \sigma_{Q_{1}}^{2}(t) \\
& +\frac{1}{2} M_{2} \Omega_{2}^{2} \sigma_{Q_{2}}^{2}(t)+\mu_{11} \sigma_{P_{1}}(t) \sigma_{Q_{1}}(t)+\mu_{22} \sigma_{P_{2}}(t) \sigma_{Q_{2}}(t) \\
& +d_{12} \sigma_{P_{1}}(t) \sigma_{P_{2}}(t)+c_{12} \sigma_{Q_{1}}(t) \sigma_{Q_{2}}(t) .
\end{aligned}
$$

Since the expectation and variances values of the coordinates and momenta decrease exponentially in time, the energy is dissipated to a minimum value. Assuming that the conditions (38) hold, the minimum value of the total energy of the system is given by the following expression:

$$
E_{\min }=E(\infty)=\hbar\left(\frac{\Omega_{1}}{2} \operatorname{coth} \frac{\hbar \Omega_{1}}{2 k T}+\frac{\Omega_{2}}{2} \operatorname{coth} \frac{\hbar \Omega_{2}}{2 k T}\right) .
$$

At zero temperature, i.e. $T=0$, the minimal energy becomes $\bar{E}_{\min }=\hbar\left(\frac{\Omega_{1}}{2}+\frac{\Omega_{2}}{2}\right)$, which corresponds to the energy of the system in its ground state. In the particular case where $\omega_{1}=\omega_{2}$ implying $\Omega_{1}=\Omega_{2}=\Omega$ and $\theta=\eta=0$, the minimum energy of the ground state can be reduced to $\bar{E}_{\min }^{\prime}=\hbar \Omega$, and we recover the ground state energy of the usual harmonic oscillator in the commutative case. Figure 5 depicts therefore, the energy with respect to the cutting frequency on one hand and with respect to the real temperature on the other hand both for different values of NC phase-space parameters.

It can be observed that, for fixed values of the temperature $T$ (the thermal bath temperature), the total energy increases quickly with the magnetic field (cutting frequency), and that under lower magnetic field effects, the total energy for

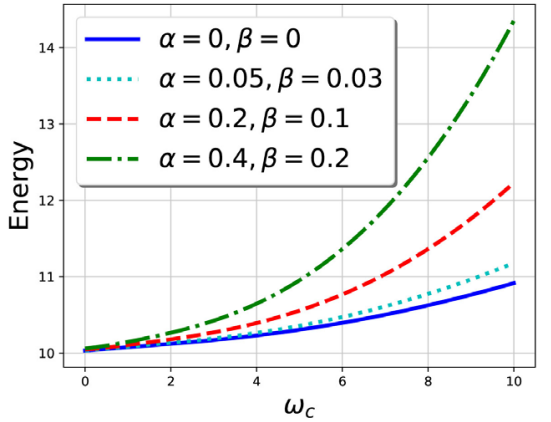

(a)

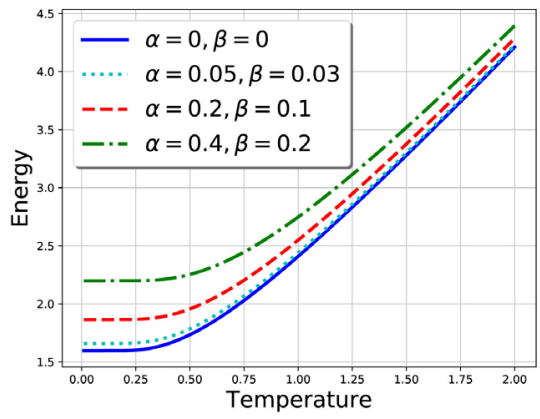

(b)

Figure 5. Energy of an harmonic oscillator in two-dimensional phase-space with respect to the cutting frequency $\omega_{c}$ and with respect to the temperature $T$ both as function of the NC phase-space parameters. 
different NC parameter values coincides. But under intense magnetic field effects, the NCty structure of the phase-space considerably affects the system since the energy increase with these effects. In addition, it is observed that, for extremely low temperature, the total energy of the system increase significantly with the NCty effects, however as the temperature increases, the energy increases too and for high temperature, the energy of the system in NC phase-space coincides with that of the system in commutative space (blue solid curve). This represents the fact that, when the temperature increases in the system, the coupling between the particle and the bath becomes considerably strong, the whole system takes a large dimension and then, the effects of quantum gravity manifest in NC phases-space are not more detectable. Similar results were found by Tchoffo et al. [26] in the case of Brownian particle in NC space. Analogically to the decoherence time scale, Figure 6 plots the energy of the system with respect to simultaneously the NC parameters in the $\mathrm{x}$ - and $\mathrm{y}$-directions, where we can easily observe that it increases with both parameters, confirming the above results.

\section{Conclusions}

In this paper, we studied decoherence of a damped anisotropic harmonic oscillator under magnetic field effects in two-dimensional NC phase-space. For this reason, the evolution of the system was studied within the framework of the Lindblad ME theory for open quantum systems, considering the general case of an environment consisting of a thermal bath at arbitrary temperature. Based on the above mentioned theory, the damping of the expectation values of coordinates and momenta was evaluated as functions of time. Then, from the ME of an NC damped anisotropic oscillator approach, the time evolution of the density matrix and the Wigner function were derived systematically. It turned out by solving these equations that:

- The solutions follow Gaussian distribution. Moreover, from the expectation values and the variances of coordinate and momenta, the total energy of the system was evaluated.

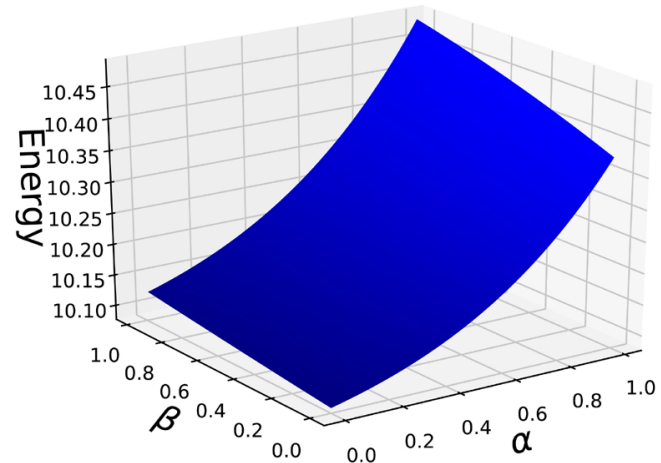

Figure 6. Energy of a harmonic oscillator in two-dimensional phase-space with respect to both the NC parameters in the $\mathrm{x}$ - and y-directions, for a fixed temperature $T=5 \mathrm{~K}$ and a fixed cutting frequency $\omega_{c}=1.5$. 
- The total energy increases significantly with the NCty effects, and as the temperature increases, the energy increases too. However, for high temperature, the energy of the system in NC phase-space coincides with that of the system in commutative space. Similar effects were observed with the magnetic field effects.

- In addition, the decoherence time scale was analytically derived, and its simulation proved that, the NCty effects present significant impact on this time scale, and thus, considerably affect decoherence in the system in the sense that, the decoherence time scale increases as the NC parameters increase. It turned out therefore that, the decoherence time scale was improved by NCty effects.

- The decoherence time was found to have a similar scale as the time after which statistic fluctuations become comparable with quantum fluctuations as expected, and the values of the scales become closer with the growth of temperature, magnetic field, and NCty effects.

\section{Conflicts of Interest}

The authors declare no conflicts of interest regarding the publication of this paper.

\section{References}

[1] Weiss, U. (2012) Quantum Dissipative Systems. World Scientific, Singapore. https://doi.org/10.1142/8334

[2] Hu, B. and Zhang, Y. (1993) Squeezed States and Uncertainty Relation at Finite Temperature. Modern Physics Letters A, 8, 3575-3584. https://doi.org/10.1142/S0217732393002312

[3] Breuer, H.-P. and Petruccione, F. (2003) Concepts and Methods in the Theory of Open Quantum Systems. In: Irreversible Quantum Dynamics, Springer, Berlin, 65-79. https://doi.org/10.1007/3-540-44874-8_4

[4] Zurek, W.H. (1982) Environment-Induced Superselection Rules. Physical Review D, 26, 1862. https://doi.org/10.1103/PhysRevD.26.1862

[5] Isar, A., Sandulescu, A. and Scheid, W. (2000) Dissipative Tunneling through a Parabolic Potential in the Lindblad Theory of Open Quantum Systems. The European Physical Journal D, 12, 3-10. https://doi.org/10.1007/s100530070035

[6] Lampo, A., Lim, S.H., Wehr, J., Massignan, P. and Lewenstein, M. (2016) Lindblad Model of Quantum Brownian Motion. Physical Review A, 94, Article ID: 042123. https://doi.org/10.1103/PhysRevA.94.042123

[7] Griessner, A., Daley, A., Clark, S., Jaksch, D. and Zoller, P. (2006) Dark-State Cooling of Atoms by Superfluid Immersion. Physical Review Letters, 97, Article ID: 220403. https://doi.org/10.1103/PhysRevLett.97.220403

[8] Diehl, S., Yi, W., Daley, A. and Zoller, P. (2010) Dissipation-Induced d-Wave Pairing of Fermionic Atoms in an Optical Lattice. Physical Review Letters, 105, Article ID: 227001. https://doi.org/10.1103/PhysRevLett.105.227001

[9] Khiari, L., Boudjedaa, T., Makhlouf, A. and Meftah, M. (2019) Coupled Oscillators in Non-Commutative Phase Space: Path Integral Approach. European Physical Journal, 134, Article No. 396. https://doi.org/10.1140/epjp/i2019-12770-3 
[10] Breuer, H.-P. and Petruccione, F. (2000) Radiation Damping and Decoherence in Quantum Electrodynamics. In: Relativistic Quantum Measurement and Decoherence, Springer, Berlin, 31-65. https://doi.org/10.1007/3-540-45369-5_3

[11] Zurek, W.H. and Paz, J.P. (1995) Quantum Chaos: A Decoherent Definition. Physica D, 83, 300-308. https://doi.org/10.1016/0167-2789(94)00271-Q

[12] Paz, J.P. and Zurek, W.H. (1993) Environment-Induced Decoherence, Classicality, and Consistency of Quantum Histories. Physical Review D, 48, 2728. https://doi.org/10.1103/PhysRevD.48.2728

[13] Lombardo, F.C. and Nacir, D.L. (2005) Decoherence during Inflation: The Generation of Classical Inhomogeneities. Physical Review D, 72, Article ID: 063506. https://doi.org/10.1103/PhysRevD.72.063506

[14] Bai, X.-F., Xin, W., Liu, X.-X., et al. (2020) Asymmetric Gaussian Confinement Potential and Decoherence Effect on Polaron in Quantum Disk with Electromagnetic Field. European Physical Journal, 135, 321. https://doi.org/10.1140/epjp/s13360-020-00321-y

[15] Isar, A. and Scheid, W. (2007) Quantum Decoherence and Classical Correlations of the Harmonic Oscillator in the Lindblad Theory. Physica A: Statistical Mechanics and Its Applications, 373, 298-312. https://doi.org/10.1016/j.physa.2006.04.065

[16] Krzywicki, A. (1993) Coherence and Decoherence in Radiation Off Colliding Heavy Ions. Physical Review D, 48, 5190. https://doi.org/10.1103/PhysRevD.48.5190

[17] Nielsen, M.A. and Chuang, I. (2002) Quantum Computation and Quantum Information. American Journal of Physics, 70, 558. https://doi.org/10.1119/1.1463744

[18] Isar, A., Sandulescu, A. and Scheid, W. (1999) Purity and Decoherence in the Theory of a Damped Harmonic Oscillator. Physical Review E, 60, 6371. https://doi.org/10.1103/PhysRevE.60.6371

[19] Davies, E.B. (1976) Quantum Theory of Open Systems. Academic Press, Cambridge.

[20] Isar, A. (1999) Uncertainty, Entropy and Decoherence of the Damped Harmonic Oscillator in the Lindblad Theory of Open Quantum Systems. Fortschritte der Physik: Progress of Physics, 47, 855-879.

https://doi.org/10.1002/(SICI)1521-3978(199909)47:7/8<855::AID-PROP855>3.0.C $\underline{\mathrm{O} ; 2-\mathrm{Z}}$

[21] Isar, A., Sandulescu, A., Scutaru, H., Stefanescu, E. and Scheid, W. (1994) Open Quantum Systems. International Journal of Modern Physics E, 3, 635-714. https://doi.org/10.1142/S0218301394000164

[22] Isar, A., Sandulescu, A. and Scheid, W. (1991) Use of Characteristic Function in Open Quantum Systems and Charge Equilibrium in Deep Inelastic Reactions. Journal of Physics G: Nuclear Physics, 17, 385. https://doi.org/10.1088/0954-3899/17/3/015

[23] Halliwell, J.J. (1989) Decoherence in Quantum Cosmology. Physical Review D, 39, 2912. https://doi.org/10.1103/PhysRevD.39.2912

[24] Dragovich, B. and Dugić, M. (2005) On Decoherence in Noncommutative Plane with Perpendicular Magnetic Field. Journal of Physics A: Mathematical and General, 38, 6603. https://doi.org/10.1088/0305-4470/38/29/014

[25] Ghorashi, S. and Harouni, M.B. (2013) Decoherence of Quantum Brownian Motion in Noncommutative Space. Physics Letters A, 377, 952-956. https://doi.org/10.1016/j.physleta.2013.02.019

[26] Tchoffo, M., Kuetche, J.C.N., Fouokeng, G.C., Afuoti, N.E. and Fai, L.C. (2014) Ki- 
nematical Brownian Motion of the Harmonic Oscillator in Non-Commutative Space. American Journal of Modern Physics, 3, 138. https://doi.org/10.11648/j.ajmp.20140303.14

[27] Santos, W.O., Almeida, G.M. and Souza, A.M. (2017) Noncommutative Brownian Motion. International Journal of Modern Physics A, 32, Article ID: 1750146. https://doi.org/10.1142/S0217751X17501469

[28] Martin, T., Armel, A.K., Germain, Y.D., Giresse, T.A. and Cornelius, F.L. (2019) Decoherence of Quantum Brownian Particle Trapped in a Penning Potential in a Non-Commutative Space. Journal of Physics Communications, 3, Article ID: 095006. https://doi.org/10.1088/2399-6528/ab2b37

[29] Chowdhury, S.H.H., Chowdhury, T.A. and Ud, A. (2020) Gauge Invariant Energy Spectra for the Noncommutative Landau Problem Subjected to an Anisotropic Harmonic Potential.

[30] Halder, A. and Gangopadhyay, S. (2017) Thermodynamics of a Charged Particle in a Noncommutative Plane in a Background Magnetic Field. International Journal of Theoretical Physics, 56, 1831-1844. https://doi.org/10.1007/s10773-017-3328-4

[31] Nath, D. and Roy, P. (2017) Noncommutative Anisotropic Oscillator in a Homogeneous Magnetic Field. Annals of Physics, 377, 115-124.

https://doi.org/10.1016/j.aop.2016.12.028

[32] Pal, S.K., Nandi, P. and Chakraborty, B. (2018) Connecting Dissipation and Noncommutativity: A Bateman System Case Study. Physical Review A, 97, Article ID: 062110. https://doi.org/10.1103/PhysRevA.97.062110

[33] Santos, J.F. and Bernardini, A.E. (2017) Quantum Engines and the Range of the Second Law of Thermodynamics in the Noncommutative Phase-Space. European Physical Journal, 132, Article No. 260. https://doi.org/10.1140/epjp/i2017-11538-1

[34] dos Santos, J., Luiz, F.S., Duarte, O.S. and Moussa, M.H. (2019) Non-Hermitian Noncommutative Quantum Mechanics. European Physical Journal, 134, Article No. 332. https://doi.org/10.1140/epjp/i2019-12738-3

[35] Bertolami, O., Rosa, J., De Aragao, C., Castorina, P. and Zappala, D. (2005) Noncommutative Gravitational Quantum Well. Physical Review D, 72, Article ID: 025010. https://doi.org/10.1103/PhysRevD.72.025010

[36] Gnatenko, K.P., Laba, H. and Tkachuk, V. (2018) Features of Free Particles System Motion in Noncommutative Phase Space and Conservation of the Total Momentum. Modern Physics Letters A, 33, Article ID: 1850131. https://doi.org/10.1142/S0217732318501316

[37] Giri, P.R. and Roy, P. (2008) The Non-Commutative Oscillator, Symmetry and the Landau Problem. European Physical Journal C, 57, 835-839.

https://doi.org/10.1140/epjc/s10052-008-0705-4

[38] Hinchliffe, I., Kersting, N. and Ma, Y. (2004) Review of the Phenomenology of Noncommutative Geometry. International Journal of Modern Physics A, 19, 179-204. https://doi.org/10.1142/S0217751X04017094

[39] Banerjee, R., Roy, B.D. and Samanta, S. (2006) Remarks on the Noncommutative Gravitational Quantum Well. Physical Review D, 74, Article ID: 045015. https://doi.org/10.1103/PhysRevD.74.045015

[40] Nair, V. and Polychronakos, A. (2001) Quantum Mechanics on the Noncommutative Plane and Sphere. Physics Letters B, 505, 267-274. https://doi.org/10.1016/S0370-2693(01)00339-2

[41] Boura, H.A., Isar, A. and Kourbolagh, Y.A. (2016) Quantum Fidelity of Two-Mode 
Gaussian States in the Two-Reservoir Model. Romanian Reports in Physics, 68, 19.

[42] Suciu, S. and Isar, A. (2016) Quantum Coherence of Two-Mode Systems in a Thermal Environment. Romanian Journal of Physics, 61, 1474.

[43] Isar, A. (2018) Generation of Quantum Steering in Gaussian Open Systems. Romanian Journal of Physics, 63, 108.

[44] Isar, A., Scheid, W. and Sandulescu, A. (1991) Quasiprobability Distributions for Open Quantum Systems within the Lindblad Theory. Journal of Mathematical Physics, 32, 2128. https://doi.org/10.1063/1.529185

[45] Isar, A. (2009) Entanglement Generation and Evolution in Open Quantum Systems. Open Systems \& Information Dynamics, 16, 205-219. https://doi.org/10.1142/S1230161209000153

[46] Schrödinger, E. (1930) Zum heisenbergschen unschärfeprinzip. Akademie der Wissenschaften.

[47] Robertson, H.P. (1929) The Uncertainty Principle. Physical Review, 34, 163. https://doi.org/10.1103/PhysRev.34.163

[48] Mihaescu, T. and Isar, A. (2017) Gaussian Quantum Steering of Two Bosonic Modes in a Thermal Environment. Quantum, 1, 3.

[49] Isar, A. (2013) Quantum Correlations of Two-Mode Gaussian Systems in a Thermal Environment. Physica Scripta, 2013, Article ID: 014035. https://doi.org/10.1088/0031-8949/2013/T153/014035

[50] Dodonov, V. and Manko, O. (1985) Quantum Damped Oscillator in a Magnetic Field. Physica A: Statistical Mechanics and Its Applications, 130, 353-366.

https://doi.org/10.1016/0378-4371(85)90111-6

[51] Agarwal, G. (1971) Brownian Motion of a Quantum Oscillator. Physical Review A, 4, 739. https://doi.org/10.1103/PhysRevA.4.739

[52] Rajagopal, A. and Rendell, R. (2001) Decoherence, Correlation, and Entanglement in a Pair of Coupled Quantum Dissipative Oscillators. Physical Review A, 63, Article ID: 022116. https://doi.org/10.1103/PhysRevA.63.022116 